\title{
Relação Entre Funcionalidade e Idade Linguística em Crianças com Síndrome de Down
}

\author{
Diogo Ribas dos Santos, ${ }^{1}$ Bianca Halberstadt, ${ }^{2}$ Anaelena Bragança de Moraes, ${ }^{2}$ \\ Ana Paula Ramos de Souza ${ }^{2}$
}

\begin{abstract}
RESUMO
Objetivo: analisar o desempenho funcional e sua correlação com a idade linguística de crianças na faixa etária de 1 a 7 anos, com diagnóstico de Síndrome de Down (SD). Método: Trata-se de um estudo transversal, descritivo, de abordagem quantitativa. Foram utilizados os instrumentos de Avaliação do Desenvolvimento da Linguagem (ADL), o Pediatric Evaluation of Disability Inventory (Pedi) e um questionário com dados sociodemográficos elaborado pelos pesquisadores. Resultados: Participaram da pesquisa 23 crianças com SD, com média de 3,6 anos de idade cronológica e média de 2,2 anos de idade linguística. As crianças apresentaram maior independência na área de mobilidade e maior atraso na área de autocuidado. Observou-se que quanto maior a idade cronológica, menores os escores em autocuidado e função social. Além disto, as crianças com maior idade linguística possuem maior mobilidade. Conclusão: Houve correlação positiva entre idade linguística, mobilidade e autocuidado.
\end{abstract}

Palavras-chave: Desenvolvimento infantil. Linguagem infantil. Síndrome de Down. Funcionalidade.

\section{RELATIONSHIP BETWEEN FUNCTIONALITY AND LANGUAGE AGE IN CHILDREN WITH DOWN SYNDROME}

\section{ABSTRACT}

Objective: To analyze the functional performance and its correlation with the linguistic age of children aged one to seven years, diagnosed with Down Syndrome (DS). Method: This is a cross-sectional, descriptive study with a quantitative approach. We used the Language Development Assessment (ADL), the Pediatric Evaluation of Disability Inventory (Pedi) and a questionnaire with sociodemographic data built by the researchers. Results: The study included 23 children with DS, with an average of 3.6 years of chronological age and an average of 2.2 years of linguistic age. The children showed greater independence in the area of mobility and greater delay in the area of self-care. It was observed that the older the chronological age, the lower the self-care and social function scores. In addition, children with higher linguistic age have greater mobility. Conclusion: There was a positive correlation between language age, mobility and self-care.

Keywords: Language development. Child language. Down Syndrome. Funcionality.

\footnotetext{
Autor correspondente. Universidade Federal de Santa Maria. Rua Tapes, Itararé, Santa Maria/RS, Brasil. CEP 97090-640. http://lattes.cnpq. br/5315350107042453. https://orcid.org/0000-0001-6914-8386. diogofono@hotmail.com

2 Universidade Federal de Santa Maria - Santa Maria/RS, Brasil.
} 


\section{INTRODUÇÃO}

Sabe-se que a Síndrome de Down (SD) é uma das causas de alteração genética e metabólica mais conhecida entre as patologias existentes na literatura. Suas características intelectuais, físicas e motoras são focos de pesquisa de diferentes áreas (REZA SUÁREZ et al., 2019). Alguns autores descrevem a SD de maneira a delinear suas características e embasar as comorbidades que acompanham a síndrome (BARATA; BRANCO, 2010), mas poucos estudos analisam os aspectos da funcionalidade desses sujeitos (MARINS; EMMEL, 2011), sobretudo a relação entre o desenvolvimento de habilidades e a autonomia que daí resulta. Coppede et al. (2012) afirmaram que crianças com SD, entre 2 e 5 anos, apresentaram baixo desempenho funcional nas áreas de mobilidade, autocuidado e função social.

Brown e Gordon (1997) citam que com frequência as crianças com maior funcionalidade estão introduzidas em ambientes familiares favoráveis aos mecanismos de resiliência, o que pode promover a conquista de sua independência. $O$ fato de a família ou cuidador não tornarem a criança dependente deles, auxilia para que ela alcance um bom desenvolvimento social e aprecie a interação com diferentes pessoas, não estando mais tão emocionalmente dependente de seus pais. Por conseguinte, é possível salientar que o desempenho funcional e cognitivo é influenciado não só pelas propriedades intrínsecas da criança, mas pelas demandas específicas de tarefas e pelas características ambientais em que ela está inserida (PINTO; NERI, 2013).

Esse comportamento familiar dependerá, ao menos parcialmente, do tipo de intervenção clínica que a criança e familiares receberam, uma vez que a valorização da participação e reflexão familiar sobre as potencialidades do filho pode facilitar a interação com a criança (SANTOS, 2015) e também a emergência de uma perspectiva de futuro para ele, favorecendo a adaptação parental e diminuindo o risco de instalação de uma psicopatologia (FRANCO, 2015).

Estudos com foco na funcionalidade (DI NUBILA; BUCHALLA, 2008) afirmam que quanto maior a autonomia da criança, melhor o seu desempenho em áreas funcionais de interação social, autonomia, mobilidade e autocuidado. Na Fonoaudiologia há poucos estudos sobre funcionalidade e linguagem (POMMEREHN; DELBONI; FEDOSSE, 2016) e não foram encontrados estudos com o tema da linguagem e funcionalidade em crianças com SD na realidade nacional. Considerando esta lacuna, este estudo objetiva ana- lisar o desempenho funcional e sua correlação com a idade linguística de crianças na faixa etária de 1 a 7 anos, com diagnóstico de SD.

\section{METODOLOGIA}

Trata-se de um estudo transversal, descritivo, de abordagem quantitativa. Foram incluídas crianças com SD na faixa etária de 1 a 7 anos de idade e seu responsável. Foram excluídas da pesquisa as crianças que possuíam outras comorbidades, como o transtorno do espectro autista, deficiência visual, entre outras, que impedissem a avaliação da linguagem.

Foram utilizados os instrumentos de Avaliação do Desenvolvimento da Linguagem (ADL), o Pediatric Evaluation of Disability Inventory (Pedi) e um questionário com dados sociodemográficos elaborado pelos pesquisadores. O ADL foi aplicado nas crianças e o Pedi e questionário com o familiar.

O ADL é utilizado para definir a idade linguística da criança, sendo aplicável dos 12 meses aos 6 anos e 11 meses. Ele é aplicado individualmente, para avaliar os domínios receptivo e expressivo da linguagem. Deste modo, foram possíveis utilizar crianças na faixa etária de 7 anos, pois o protocolo de avaliação permite avaliar cada criança a partir de seis meses inferiores a sua idade cronológica. Se a criança não conseguisse realizar a atividade correspondente com sua idade, era realizado um rastreio com protocolos de idade inferior a dela até uma idade mínima que pudesse desempenhar adequadamente. A partir da aplicação foi identificada a idade linguística (IL), idade de recepção $(I R)$ e de expressão (IE). Esses dados foram comparados descritivamente à idade cronológica (ICr) (MENEZES, 2003).

O Pedi é adotado para avaliar quantitativamente a capacidade funcional da criança e a independência para realizar as atividades de autocuidado, mobilidade e função social. A aplicação do Pedi é feita por meio de uma entrevista com o familiar ou responsável pela criança (MANCINI, 2005). Em cada grupo etário o intervalo de normalidade compreende um escore normativo (EN) entre 30 e 70. Quando inferior a 30 representa atraso ou desenvolvimento significativamente inferior (ALEGRETTI; MANCINI; SCHWARTZMAN, 2002).

A coleta de dados foi realizada nos locais que proporcionam atendimento a crianças com deficiência intelectual na região central do Estado do Rio Grande do Sul. A partir da autorização do Comitê de Ética e das instituições participantes, os pesquisadores entraram em contato com os possíveis participantes. Após 
o aceite da família, foram estipulados hora e local para a coleta de dados, em uma sala disponibilizada pelas instituições.

Esses dados foram digitados em uma planilha eletrônica do tipo Excel e convertidos para o aplicativo computacional Statistica 9.1, para a realização das análises estatísticas que constaram de análise descritiva (percentuais, média, mediana, valor mínimo, valor máximo, desvio padrão e coeficiente de variação) e teste de correlação de Spearman para as variáveis idade linguística, idade cronológica e funcionalidade da criança. Foi considerado o nível de significância de $5 \%$.

A presente pesquisa faz parte do projeto intitulado "Desenvolvimento infantil e sua relação com adaptação parental à incapacidade do filho: análise da associação com aspectos sociodemográficos e oportunidades clínicas e educacionais", aprovado pelo Comitê de Ética em Pesquisa de Instituição de Ensino Superior de uma cidade de porte médio do Rio Grande do Sul, sob número do CAEE: 02235218.9.0000.5346. R espeita as normas e diretrizes regulamentadoras para pesquisa com seres humanos que estão na Resolução 466/12 do Conselho Nacional de Saúde, garantindo sigilo e privacidade da identidade dos sujeitos. Houve o esclarecimento dos objetivos e procedimentos às famílias que assinaram o Termo de Consentimento Livre e Esclarecido após terem aceitado participar da pesquisa.

\section{RESULTADOS}

Participaram da pesquisa 23 crianças com SD, 11 do sexo feminino $(47,8 \%)$ e 12 do sexo masculino $(52,2 \%)$, observando-se que grande parte possui renda familiar inferior a $\mathrm{R} \$ 2.000,00$, conforme pode-se visualizar na Tabela 1.
Tabela 1 - Análise descritiva da variável renda familiar

\begin{tabular}{lc}
\hline Renda em reais & $\%$ \\
\hline Até 1.000 & 17,4 \\
De 1.000 a 2.000 & 39,1 \\
De 2.000 a 3.000 & 13,0 \\
De 3.000 a 4.000 & 4,3 \\
De 4.000 a 5.000 & 8,7 \\
De 5.000 a 6.000 & 4,3 \\
De 6.000 a 7.000 & 13,0 \\
\hline
\end{tabular}

Fonte: Elaborada pelos autores.

Em média os sujeitos da pesquisa apresentaram 3,6 anos de idade cronológica e idade linguística média de 2,2 anos, conforme se visualiza na Tabela 2 . Na mesma tabela pode-se observar o coeficiente de variação entre idade linguística e idade cronológica. As variáveis testadas encontraram média de 1,4 ano e mediana de 0,7 ano, com valor mínimo de zero ano e máximo de 5,6 anos. 0 desvio padrão encontrado foi de 1,6 ano, assim, o coeficiente de variação é igual a $114,3 \%$, demonstrando que existe muita variabilidade entre as médias.

Em relação aos escores de autocuidado, mobilidade e função social, é possível entender que $50 \%$ das crianças têm a diferença de 8,4 meses de idade ou menos e as outras $50 \%$ apresentaram 8,4 meses ou mais, demonstrando harmonia entre os valores encontrados.

Os resultados evidenciaram que há diferença significativa $(p=0,000)$ entre as médias de idade linguística e idade cronológica.

Em relação às variáveis de funcionalidade constante no protocolo Pedi e à idade linguística encontrada por meio da avaliação do ADL, verificou-se a corre-

Tabela 2 - Medidas descritivas da idade cronológica, idade linguística, escores do Pedi

\begin{tabular}{lcccccc}
\hline Variáveis & Média & Mediana & Mínimo & Máximo & $\begin{array}{c}\text { Desvio } \\
\text { padrão }\end{array}$ & $\begin{array}{c}\text { Coeficiente de } \\
\text { variação\% }\end{array}$ \\
\hline Idade cronológica & 3,6 & 3,0 & 1,1 & 7,0 & 1,9 & 53,6 \\
Idade linguística & 2,2 & 1,4 & 1,0 & 6,50 & 1,5 & 66,5 \\
Escore autocuidado & 22,0 & 16,7 & 10,0 & 58,4 & 15,1 & 68,5 \\
Escore mobilidade & 28,9 & 25,10 & 10,0 & 56,2 & 16,7 & 57,8 \\
Escore função social & 27,7 & 18,4 & 10,0 & 63,1 & 19,6 & 70,7 \\
\hline
\end{tabular}


lação entre os escores de autocuidado, mobilidade e função social com idade linguística e idade cronológica de cada criança, conforme se observa na Tabela 3.

Tabela 3 - Correlação entre Pedi, idade cronológica, idade linguística e renda familiar

\begin{tabular}{lcc}
\hline Variáveis & $\mathbf{R}$ & $\mathrm{P}$ \\
\hline Idade cronológica x escore autocuidado** $^{*}$ & $-0,81$ & 0,000 \\
Idade cronológica x escore função social $^{* *}$ & $-0,55$ & 0,007 \\
Idade cronológica x escore mobilidade & 0,11 & 0,602 \\
Idade linguística x escore mobilidade** & 0,73 & 0,000 \\
Idade linguística x escore autocuidado & $-0,37$ & 0,080 \\
Idade linguística x escore função social & $-0,08$ & 0,688 \\
\hline
\end{tabular}

$r=$ Coeficiente de Spearman; ${ }^{* *}$ Valores com correlação significativa. Fonte: Elaborada pelos autores.

Na Tabela 3 observa-se que quanto maior a idade cronológica menor os escores em autocuidado (correlação forte) e função social (correlação moderada, mas significativa), ou seja, as crianças mais velhas apresentam maior atraso em autocuidado e em função social em relação às mais jovens. Já sobre a idade linguística houve uma correlação positiva em relação à mobilidade, ou seja, crianças com maior idade linguística possuem maior mobilidade.

\section{DISCUSSÃO}

Considerando os resultados da pesquisa foi possível verificar que a diferença entre idade cronológica e idade linguística foi de 1 ano e 3 meses. Para Ciciliato, Zilotti e Mandra (2010), as crianças com SD apresentam atrasos significativos de linguagem, contudo as habilidades são adquiridas na mesma sequência observada em crianças com o desenvolvimento típico (CHAN; IACONO, 2001; O'TOOLE; CHIAT, 2006).

$O$ desenvolvimento adequado da linguagem oral na infância é fundamental para que a criança se socialize (SHERIDAN; GJEMS, 2017), contribuindo nas relações pessoais, na inserção e participação em atividades e práticas sociais (LIMA; DELGADO; CAVALCAN$T E, 2017)$, além de favorecer o desenvolvimento de outras habilidades (SHERIDAN; GJEMS, 2017).

Sabe-se que na literatura existem diversos estudos acerca das dificuldades de linguagem das crianças com SD, os quais destacam a importância da intervenção precoce para minimizar o atraso inicial na aquisição da linguagem, para facilitar a ampliação do repertório linguístico e reduzir os atrasos relacionados ao déficit intelectual (PEREIRA; OLIVEIRA, 2015). Neste sentido, as alterações no desenvolvimento da linguagem são vistas com preocupação por profissionais da saúde e de todas as áreas que atuam com desenvolvimento infantil (BETTIO; BAZON; SCHIMDT, 2019).

Além do atraso na linguagem, as crianças desta pesquisa apresentaram atraso na área de autocuidado e função social. Este fato pode em alguns momentos estar associado ao processo de privação e/ou superproteção parental. A falta de convicção de que a criança tem capacidade de ser independente e ter autonomia é uma dificuldade que os pais, com frequência, enfrentam. A potencialidade de cada criança para aprender a interagir em condições satisfatórias em seu meio familiar e em seu ambiente social pode ser estimulada ou inibida pela atitude das pessoas que a rodeiam (MARTIN; BUENO, 2003).

Resultados semelhantes sobre a funcionalidade infantil foram encontrados por Gomes et al. (2017). Os autores fizeram um estudo em Goiânia para avaliar o desempenho funcional de 28 crianças com 5 e 6 anos de idade (14 com SD e 14 com desenvolvimento motor típico), utilizando o Pedi. As crianças com SD apresentaram desempenho funcional inferior em relação àquelas com desenvolvimento típico da mesma idade, principalmente nas habilidades funcionais em função social e na assistência do cuidador em autocuidado.

As habilidades funcionais de autocuidado, avaliadas pelo Inventário Pedi (MANCINI, 2005), demonstraram que a pessoa com SD apresenta atrasos no desempenho funcional, mesmo em idades superiores às que já haviam sido estudadas por Mancini et al. (2003), que abordaram a faixa etária dos 2 e 5 anos, uma vez que a maioria dos sujeitos apresentou um rendimento abaixo de $100 \%$ para a área de autocuidado (SOUZA et al., 2012). Os dados desses estudos confirmam os resultados desta pesquisa.

Observa-se, na Tabela 3, que houve correlação significativa entre os resultados entre idade cronológica e funcionalidade (autocuidado e função social). Eles demonstraram que crianças mais velhas podem ter maiores exigências em habilidades de autocuidado e na função social a ponto de fazerem emergir as diferenças entre elas e a criança em desenvolvimento típico. A cada nova fase de desenvolvimento, a criança necessita aprender habilidades crescentemente mais complexas ligadas a um repertório previsto para sua faixa etária (PAZIN; MARTINS, 2007).

De acordo com os resultados evidenciados neste estudo, o escore de mobilidade é o mais aproximado da faixa de normalidade, ou seja, não é considerado como um problema para as crianças com SD da 
pesquisa. A idade linguística quando testada com os escores de funcionalidade da Pedi, evidenciou que a criança com maior mobilidade é aquela com melhor desenvolvimento global. Assim sendo, parece ter se evidenciado que a idade linguística associada aos limiares de mobilidade apresentou maior correlação quando está ligada ao autocuidado e função social. Essa correlação pode estar associada ao fato de ter a inserção de terapeutas fonoaudiólogos e fisioterapeutas em equipes de intervenção precoce com maior frequência. As questões de autocuidado e função social, as quais não apresentaram correlação significativa, salientaram a importância do trabalho do terapeuta ocupacional em equipes de intervenção com o objetivo de prevenir a presença de alterações e dificuldades de interação social.

\section{CONCLUSÃO}

Conclui-se que houve correlação positiva entre idade linguística e funcionalidade. A funcionalidade piora com o aumento da idade cronológica em virtude do aumento de exigências das crianças.

À vista do que foi encontrado, sugere-se que outras pesquisas no campo da funcionalidade e linguagem sejam realizadas, com uma população maior, para que se possa dar continuidade aos achados neste estudo.

\section{REFERÊNCIAS}

ALLEGRETTI, Ana Luiza C.; MANCINI, Marisa C.; SCHWARTZMAN, José Salomão. Estudo do desempenho funcional de crianças com paralisia cerebral diparética espástica utilizando o Pediatric Evaluation of Disability Inventory (PEDI). Temas Desenvolv., v. 11, n. 64, p. 5-11, 2002.

BARATA, Lívia Fernandes; BRANCO, Anete. Os distúrbios fonoarticulatórios na síndrome de Down e a intervenção precoce. Revista Cefac, v. 12, n. 1, p. 134-139, 2010.

BETTIO, Claudia Daiane Batista; BAZON, Marina Rezende; SCHMIDT, Andréia. Fatores de risco e proteção para atraso no desenvolvimento da linguagem. Psicol. Estud., Maringá, v. 24, e41889, 2019.

BROWN, M.; GORDON, W.A. Impact of impairment on activity patterns of children. Arch Phys Med Rehabil., 68, p. 828-832, 1997.

CHAN, Jeffrey, IACONO, Teresa. Gesture and word production in children with Down Syndrome. Augment Altern Commun., 17(2), p. 73-87, 2001.

CICILIATO, Mariane Nardezi; ZILOTTI, Daiana Camargo; MANDRA, Patrícia Pupin. Caracterização das habilidades simbólicas de crianças com síndrome de Down. Rev. Soc. Bras. Fonoaudiol., São Paulo, v. 15, n. 3, p. 408-414, 2010.
COPPEDE, Aline Cirelli et al. Desempenho motor fino e funcionalidade em crianças com Síndrome de Down. Revista de Fisioterapia e Pesquisa, v. 19, n. 4, p. 363-368, 2012.

DI NUBILA, Heloisa Brunow Ventura; BUCHALLA, Cassia Maria. O papel das Classificações da OMS-CID e CIF nas definições de deficiência e incapacidade. Revista Brasileira de Epidemiologia, v. 11, n. 2, p. 324-335, 2008.

FRANCO, Vitor. Paixão-dor-paixão: pathos, luto e melancolia no nascimento da criança com deficiência. Revista Latino-Americana de Psicopatologia Fundamental [on-line], 18 (2), p. 204-220, 2015.

GOMES, Ueliton dos Santos et al. Desempenho funcional de crianças com Síndrome de Down com seis anos de idade. Evs, Goiânia, v. 44, 28-36, 2017.

LIMA, Ivonaldo Leidson Barbosa; DELGADO, Isabelle Cahino, CAVALCANTE, Marianne Carvalho Bezerra. Desenvolvimento da linguagem na síndrome de Down: análise da literatura Distúrb. Comum, São Paulo, 29(2), p. 354-364, jun. 2017.

MANCINI, Marisa Cotta et al. Comparison of functional performance among children with Down syndrome and children with age-appropriate development at 2 and 5 years of age. Arquivos de Neuro-Psiquiatria, v. 61, n. 2B, p. 409-415, 2003.

MANCINI, Marisa Cotta. Inventário de avaliação pediátrica de incapacidade (PEDI). Belo Horizonte: Editora UFMG, 2005.

MARTíN, M. B.; BUENO, S. T. Deficiência visual - aspectos psicoevolutivos e educacionais. Tradução Magali Lourdes Pedro. São Paulo: Santos Livraria, 2003.

MARINS, Simone Cristina Fanhani; EMMEL, Maria Luisa G. Formação do terapeuta ocupacional: acessibilidade e tecnologias/capacitation of the occupational therapist: accessibility and technologies. Cadernos Brasileiros de Terapia Ocupacional, v. 19, n. 1, 2011.

MENEZES, Maria Lúcia Novaes. A construção de um instrumento para avaliação do desenvolvimento da linguagem $A D L$ : idealização, estudo piloto para padronização e validação. 2003. Tese (Doutorado) - Fundação Oswaldo Cruz, Rio de Janeiro, RJ, 2003.

O'TOOLE, Ciara; CHIAT, Shula. Symbolic functioning and language development in children with Down syndrome. International Journal of Language \& Communication Disorders, v. 41, n. 2, p. 155-171, 2006.

PAZIN, Ana Carolina; MARTINS, Marielza R. Ismael. Desempenho funcional de crianças com Síndrome de Down e a qualidade de vida de seus cuidadores. Rev. Neurocienc., v. 15, n. 4, p. 297-303, 2007.

PEREIRA, Letícia Viana; OLIVEIRA, Erika Maria Parlato. Influência do entorno familiar no desempenho comunicativo de crianças com síndrome de Down. Revista Cefac, v. 17, n. 1, p. 177-183, 2015. 
PINTO, Juliana Martins; NERI, Anita Liberalesso. Doenças crônicas, capacidade funcional, envolvimento social e satisfação em idosos comunitários: estudo fibra. Ciênc. Saúde Coletiva, Rio de Janeiro, v. 18, n. 12, p. 3.449-3.460, dez. 2013.

POMMEREHN, Jodeli; DELBONI, Miriam Cabrera Corvelo; FEDOSSE, Elenir. Classificação internacional de funcionalidade, incapacidade e saúde e afasia: um estudo da participação social. Codas, São Paulo, v. 28, n. 2, p. 132-140, abr. 2016.

REZA SUÁREZ, Lilian et al. Síndrome de Down e seu impacto no desempenho acadêmico. Caribe de Ciências Sociais, v. 7, n. 31, p. 122-133, 2019.

SANTOS, J. B. G. A influência da psicomotricidade no processo de aprendizagem em crianças com Síndrome de Down. Fiep Bulletin, v. 85, 2015.

SHERIDAN, Sonja; GJEMS, Liv. A pré-escola como uma arena para o desenvolvimento do conhecimento dos professores sobre o aprendizado de idiomas das crianças. Revista de Educação Infantil, v. 45, n. 3, p. 347-357, 2017.

SOUZA, Alice Bernardi et al. Síndrome de Down: correlação entre o desempenho funcional com a força de preensão palmar e a destreza manual. Fisioterapia Brasil, 13, p. 22327, 2012. 